\title{
DIVERSIDAD Y RETOS EN LA DESCENTRALIZACIÓN MUNICIPAL EN COLOMBIA*
}

\author{
John Dugas, Angélica Ocampo, Luis Javier Orjuela y Germán Ruiz**
}

Fecha de recepción: octubre de 1999

Fecha de aceptación y versión final: diciembre de 1999

Resumen: Este artículo tiene como objetivo evaluar cuál fue el impacto real de la reforma descentralizada de la vida municipal colombiana despues de un periodo de casi cuatro años de haberse aplicado dichas reformas. La evaluación se fundamenta en un estudio empírico sobre las expectativas y percepción de los ciudadanos acerca de los resultados de la reforma; dicho estudio fue realizado en diez municipios del país. Los resultados muestran una gran heterogeneidad en las percepciones de los encuestados las cuales oscilan desde el elogio a los avances en la participación democrática y la autonomía municipal hasta las críticas a la falta de desarrollo de la reforma municipal debido al desfase existente entre los recursos transferidos a los municipios y las nuevas responsabilidades. Por consiguiente, las preguntas que a manera de hipótesis aborda este artículo, tienen que ver con las razones por las cuales se produjeron resultados tan disímiles y con la identificación de la clave para entender la heterogeneidad del proceso de descentralización.

Palabras clave: Reforma descentralizada, heterogeneidad perceptiva, participación democrática, autonomía municipal.

Abstract: This article evaluates the consequences of Local Decentralization in several municipalities of Colombia four years after the process began. The evaluation is based on the emprirical results drawn from two interviews about the expectations and perceptions that citizens had about the reforms. The results show great heterogeneity in these perceptions that range from favorable opinions about the advacements of democratic participation and municipal autonomy to sharp criticisms to the low pace of the process due to the existing gap between the scarcety of needed economic resources and the increase of responsabilities. The questions or hypotheses posed in this article relate to the reasons and keys to understand such heterogeneity.

Key words: Decentralized reform, perceptive heterogeneity, democratic participation, municipal autonomy.

\section{INTRODUCCION E HIPÓTESIS}

Este capítulo tiene como objetivo teorizar sobre los efectos de la descentralización municipal en Colombia a partir de los resultados de las dos encuestas realizadas en diez municipios de Colombia los cuales pertenecen a los Departamentos de Cundinamarca y la Costa Atlántica que han tenido procesos históricos y políticos muy diferentes'.

La conclusión más llamativa del estudio es que en los dos primeros años de la reforma municipal los resultados fueron muy heterogéneos (Dugas, Ocampo, Orjuela, y Ruiz, 1992: 87-120). Esto se deduce tanto de la opinión de los ciudadanos encuestados como de las observaciones del trabajo de campo. El análisis empírico sugiere que, bajo el liderazgo de los primeros alcaldes populares, los diez municipios tomaron diversos caminos en el manejo de sus nuevos recursos y responsabilidades. A partir de esta reflexión podemos hacer extensivo este análisis a todos los municipios del país. En efecto, la experiencia de los más de mil municipios de Colombia cabe dentro de un espectro muy amplio, que va desde resultados bastante negativos hasta desenlaces muy favorables.

Vale la pena destacar que esta conclusión difiere de otros análisis que perciben los resultados de la reforma municipal en términos globales. Por ejemplo, algunos estudios la evalúan negativamente, debido al desfase existente entre los recursos transferidos a los municipios y las nuevas respon-

* El presente artículo fue publicado por los mismos autores en el libro, Los caminos de la descentralización: diversidad y retos de la transformación municipal. Bogotá : Universidad de Los Andes. 1992. Hemos creído conveniente incluirlo con el permiso de los autores dada la escasez de análisis teóricos realizados a partir de estudios empíricos exhaustivos, como lo es este caso.

** John Dugas es profesor de Ciencia Política en Kalamazoo College (Michigan, EEUU) y ha sido profesor visitante en dos ocasiones en la Universidad de los Andes (Bogotá, Colombia).凶jdugas@kzoo.edu

Luis Javier Orjuela es profesor asociado del Departamento de Ciencia Politica de la Universidad de los Andes (Bogotá, Colombia). Licenciado en Derecho de la Universidad Santo Tomas de Bogotá; Magister en Administración Pública de la Escuela Superior de Ciencias Administrativas de Speyer (Alemania); Master (MA) en Ciencia Política de la Universidad Internacional de la Florida; candidato a Ph.D en Ciencia Política de la misma Universidad. $\triangle$ lorjuela@uniandes.edu.co

German Ruiz es Ph. D. en Economía, Universidad de Texas en Austin (Estados Unidos). Magister en Planeación, Universidad de Gales (Gran Bretaña). Politólogo, Universidad de los Andes (Bogotá, Colombia). Director del Centro de Apoyo Académico al Legislativo. Profesor Asociado, Ciencia Política, Universidad de los Andes. $\bowtie$ gerviz@uniandes.edu.co

1. La primera encuesta fue realizada a 1838 personas durante la campaña electoral de las primeras elecciones descentralizadas municipales de 1988 y la segunda fue levantada dos años mas tarde, en 1990, a un muestreo de 1793 personas. 
sabilidades. Si bien es cierto que la falta de recursos dificultó el desarrollo de la reforma municipal, esta limitación no impidió que muchos municipios lograran resultados positivos. Por otro lado, la reforma municipal ha sido elogiada por sus avances en materias como la participación democrática y la autonomía municipal, pero no se menciona que en muchas localidades los logros de este proceso han sido prácticamente nulos.

Evidentemente, la reforma descentralista significó un cambio profundo en la vida municipal, con nuevas responsabilidades y nuevos recursos para ser manejados por la administración local. Como ya se mencionó, después de dos años algunos municipios alcanzaron resultados bastante positivos, como el mejoramiento de la infraestructura física y administrativa, también de la situación económica, el saneamiento de las prácticas clientelistas y de corrupción y un intento por ampliar la participación ciudadana. Sin embargo, en otros municipios los problemas preexistentes de infraestructura, de corrupción administrativa y de clientelismo inclusive se exacerbaron. También hubo municipios donde no se presentó ningún cambio significativo. La pregunta es, entonces, ¿por qué la reforma municipal produjo resultados tan disímiles?

¿Cuál es la clave para entender la heterogeneidad del proceso de descentralización?

La hipótesis de nuestro estudio es que el éxito o fracaso relativo de la reforma municipal depende de ciertos factores propios de cada municipio. Estos factores son de naturaleza "estructural" y "coyuntural". En términos generales, se puede afirmar que el efecto de la combinación específica de los factores estructurales y coyunturales sobre el manejo de las nuevas responsabilidades y los nuevos recursos fue lo que determinó el impacto positivo o negativo de la reforma en cada municipio.

Los factores "estructurales" de un municipio son aquellas condiciones de carácter semipermanente; es decir, estructuras que solo se transforman a través de un proceso relativamente largo y difícil. Tales estructuras incluyen la infraestructura física y administrativa del municipio, su base económica y su tradición cívica. Todos estos factores tuvieron una gran incidencia en el desarrollo de la reforma municipal. En efecto, la posibilidad de alcanzar resultados positivos era mucho mayor en los municipios que ya contaban con una buena infraestructura física y administrativa, una fuerte base económica y una larga tradición cívica. Por el contrario, un municipio caracterizado por una infraestructura física y administrativa deficiente, una débil base económica, una fuerte tradición clientelista y bajos niveles de participación, afrontó desde el principio un conjunto de obstáculos que dificultaron el buen manejo de las responsabilidades y los recursos previstos en la reforma municipal.

Los factores "coyunturales" se refieren a los aspectos de naturaleza transitoria de vida municipal. En efecto, son sujetos a cambiar por lo menos en cada elección municipal.

Entre estos factores están la personalidad y capacidad de liderazgo del alcalde, sus propuestas concretas, la composición del Consejo municipal y las relaciones interinstitucionales entre el alcalde y el Consejo, y entre éstos, el
Departamento y la Nación. Difícilmente se pueden medir tales elementos con las herramientas de las ciencias sociales. No obstante, como en el caso de las estructuras municipales, los factores coyunturales jugaron un papel importante en determinar el relativo éxito o fracaso de la reforma municipal. Es claro, por ejemplo, que un municipio con un alcalde dinámico y capaz, con propuestas realistas y con buenas relaciones con las diversas instancias político - administrativas, tenía una mayor posibilidad de obtener buenos resultados que un municipio carente de estas condiciones.

Ni los factores estructurales, ni los factores coyunturales, por sí solos, pueden explicar la trayectoria de la reforma en cada caso. Sin embargo, al mirar estos factores en conjunto se puede entender el curso que ella tomó en cada municipio. En términos generales, se puede concluir que los municipios con una combinación de factores estructurales y coyunturales "favorables", tendieron a mostrar resultados positivos en cuanto a la reforma municipal. Al otro extremo, los municipios que iniciaron la reforma combinando factores estructurales y coyunturales "desfavorables" tendieron al fracaso en sus intentos de cambio. Tal vez las situaciones más interesantes se presentaron en los municipios donde los aspectos "favorables" y "desfavorables" se mezclaban. El resultado en estos casos tendía a ser el estancamiento de la reforma y, en algunos casos, un retroceso en la vida municipal.

En síntesis, el planteamiento básico es que la compleja combinación de los factores estructurales y coyunturales en cada municipio determinó el grado en que la administración local logró resultados positivos o negativos en el manejo de los recursos y las responsabilidades de la reforma municipal.

A continuación se examinarán los factores estructurales y coyunturales más sobresalientes, y se ilustrará sobre cómo estos factores se combinaron para determinar el resultado de la reforma en algunos de los municipios de nuestro estudio.

\section{LOS FACTORES ESTRUCTURALES}

Los factores estructurales incidieron de manera muy directa sobre los resultados de la reforma municipal. Si bien un municipio dotado con estructuras adecuadas no tenía asegurado el éxito de la reforma municipal, sí partía de una base mucho más propicia para alcanzar tal resultado. $\mathrm{Al}$ mismo tiempo, un municipio con unas estructuras precarias o inexistentes tenía que superar grandes obstáculos para lograr un desempeño aceptable. Los factores estructurales pertinentes a la vida municipal son diversos, pero pueden resumirse en tres: la infraestructura municipal, la base económica y la tradición cívica del municipio.

\section{La infraestructura municipal}

La infraestructura municipal está compuesta por: 1) la infraestructura física y 2) la infraestructura administrativa.

Por infraestructura física se entiende tanto la disponibilidad como el estado físico de los establecimientos y los servicios públicos. Entre ellos, los más importantes son: 1) 
los servicios de acueducto, alcantarillado, plazas de mercado, mataderos públicos y recolección de basuras; 2) los centros y puestos de salud y hospitales locales; 3 ) los planteles escolares; 4) el servicio de energía eléctrica; 5) el servicio de telefonía; 6) las instalaciones deportivas y recreativas; 7) las vías y los parques urbanos; 8) los caminos vecinales, y 9) los puertos fluviales.

Todos estos componentes afectan el desarrollo de la vida municipal, aun cuando no son manejados directamente por el gobierno local. No obstante, a partir de la reforma muchos de estos servicios pasaron a ser responsabilidad directa del municipio. Específicamente, el decreto 77 de 1987 estableció que "corresponde a los municipios y al Distrito Especial de Bogotá la prestación de los servicios de agua potable, saneamiento básico, matadero público, aseo público y plazas de mercado" ${ }^{2}$. El decreto también le asigna al municipio (entre otras competencias) la construcción, dotación y mantenimiento de los centros de salud y hospitales locales, y de los planteles escolares e instalaciones deportivas y de recreación ${ }^{3}$. Hay componentes de la infraestructura física que muchos municipios no manejan directamente, por ejemplo, los servicios de telefonía y energía eléctrica. Sin embargo, la ley 49 de 1987 otorgó al alcalde municipal la función de "coordinar y supervisar los servicios que presten en el municipio entidades nacionales o departamentales ${ }^{4}$.

Es de notar que las fallas en la infraestructura física de los municipios del país conforman uno de los principales problemas del desarrollo local. Por ejemplo, se estima que la cobertura nacional de agua potable sólo llega al $57 \%$ de la población, mientras la de alcantarillado apenas alcanza al 41\% de los ciudadanos (Zapata, 1991: 184). El estado de la infraestructura física está claramente relacionado con factores como el tamaño del municipio y la zona (rural o urbana) y región donde está ubicado. Sin duda, los municipios pequeños y rurales se enfrentan a los problemas más graves de infraestructura física. Esto se nota, por ejemplo, en los municipios con menos de 30.000 habitantes, que tienen un promedio de $42 \%$ de cobertura en los servicios, mientras otros tienen una cobertura de más del 70\% (Blanquer, 1991: 121). Por otro lado, la región en la que esté ubicado el municipio también influye en el nivel de infraestructura. En Antioquia, por ejemplo, es notable la cobertura de agua potable en las zonas rurales, debido en gran medida a los esfuerzos departamentales (García, 1991: 9-A).

El estado de la infraestructura física al comenzar el primer período del alcalde popular afectó la reforma municipal de dos maneras. En primer lugar, el estado inicial de cada componente de la infraestructura física influyó en el grado de desarrollo posterior de éste: por ejemplo, un municipio que contaba con una red de acueducto aceptable encontraba menos dificultades para mejorar y expandir su cobertura que uno que comenzaba la reforma municipal con serios problemas en este campo. Además, en el primer caso la municipalidad tenía mayores posibilidades de invertir sus limitados recursos en otros rubros de la infraestructura física.

En segundo lugar, el estado inicial de la infraestructura física en su conjunto afectó el resultado de la reforma municipal. En este sentido, un municipio que partió con deficiencias serias en casi todos los establecimientos y servicios públi- cos se encontraba ante una disyuntiva: o bien mantenía esta grave situación, dada la escasez de los recursos y la dificultad de decidir en qué campo invertirlos, o bien ignoraba algunas necesidades sentidas del municipio, a fin de poder sacar adelante la construcción, mejoramiento o extensión de otro servicio prioritario para el bienestar de la comunidad.

Por todo lo anterior, es claro que un alcalde popular, que comenzó su gobierno con una adecuada infraestructura física, se encontraba en condiciones más favorables para asumir el control sobre los establecimientos y servicios públicos, mantenerlos, mejorarlos y extender su cobertura.

El otro componente importante de la infraestructura municipal es el que hemos denominado la infraestructura administrativa; esto es el conjunto de factores que influyen directamente sobre la administración de las funciones y los recursos del gobierno municipal. Aquí se incluye: 1) la organización de la administración municipal, 2) su capacidad administrativa, y 3) las técnicas administrativas utilizadas.

La adecuada organización del gobierno local fue fundamental para el logro de una buena gestión municipal en los dos primeros años de la labor del alcalde popular. La conformación de la estructura municipal le corresponde expresa y exclusivamente al municipio. En este sentido, la Constitución de 1991 le confiere al Consejo Municipal la responsabilidad de "determinar la estructura de la administración municipal y las funciones de sus dependencias" ${ }^{5}$. Los primeros alcaldes elegidos popularmente heredaron de sus antecesores una estructura municipal, con la cual o bien tuvieron que trabajar, o bien intentaron transformar. Si el alcalde ya contaba con una estructura lógica y ajustada a las necesidades del gobierno local tenía cierta ventaja para poner en marcha la reforma municipal. Al contrario, si la estructura era anticuada e ineficiente tenía que enfrentar varios obstáculos para llevar a cabo una buena gestión.

El segundo elemento de la infraestructura administrativa del municipio es su capacidad administrativa. Con este término hacemos referencia a los recursos humanos de la administración pública. Tres aspectos son esenciales: la cantidad de funcionarios, su estabilidad laboral y su capacitación para ejercer las funciones que les corresponden. De hecho, obstáculos de diversa índole dificultan una buena gestión administrativa. En cuanto al número de funcionarios, los problemas surgen, o bien por ser insuficientes, debido a la falta de presupuesto, o bien por ser demasiados, como consecuencia del clientelismo local. La falta de estabilidad laboral se relaciona igualmente con el clientelismo ya que muchos nombramientos obedecen a recomendaciones e influencias. De tal manera, la suerte de un jefe político y el comportamiento del funcionario respecto a su jefe influyen de manera desmesurada en la estabilidad laboral de la administración local. La inadecuada capacitación de los servidores públicos constituye también un problema en muchos municipios; éste

2. Artículo $1^{\circ}$ del decreto 77 de 1987

3. Artículo 18 y 23 del decreto 77 de 1987

4. Artículo $5^{\circ}$ de la ley 49 de 1987.

5. En la Constitución de 1886 esta responsabilidad estaba plasmada en el numeral 3 del artículo 197. En la de 1991 se consagra en el numeral 6 del artículo 313 . 
se volvió más agudo al tener que asumir la dirección y administración de los servicios públicos previstos en la reforma municipal. Además, la capacidad administrativa se ve afectada por los nexos entre el gobierno local y los sindicatos de trabajadores del municipio y sus entidades descentralizadas, en tanto que éstos actúen como apoyo o como obstáculo para una buena gestión municipal. Finalmente, como veremos más adelante, el grado de corrupción existente en el gobierno local incide en la capacidad administrativa.

Un tercer elemento infraestructura municipal que influyó en la efectividad y alcances de la reforma fue el tipo de técnicas administrativas utilizadas. El empleo de técnicas modernas de contabilidad de registros y archivos, de planeación, y de la informática es fundamental y creciente en importancia según el tamaño del municipio. Todas las municipalidades que introdujeron métodos avanzados de administración derivaron enormes beneficios de ello. Esto fue especialmente importante para la adecuada recolección de los impuestos municipales previstos en la ley 14 de 1983. Por ejemplo, si el municipio contaba con archivos actualizados o con un banco de datos computarizado sobre la actividad económica local podía ser muy eficiente en la recolección del impuesto de Industria y Comercio. En fin, cualquier municipio con técnicas avanzadas y adecuadas de informática, registros y archivos, contabilidad y planeación, ofrecía buenas condiciones para un óptimo desarrollo de la reforma municipal.

\section{La base económica}

La base económica es el factor estructural de mayor rigidez. Difícilmente se puede cambiar en el corto plazo la estructura económica de un municipio. En principio, la base económica consiste en la composición, tamaño y estabilidad de la actividad económica municipal. Un cuarto elemento importante es el empleo de los recursos humanos. Este último es fundamental dado que pueden existir municipios con una fuerte actividad económica pero que al mismo tiempo tienen un alto nivel de desempleo.

Es de notar la falta de datos confiables sobre los ingresos per cápita y el desempleo en los municipios del país. Sin embargo, al analizar unos datos regionales se pueden trazar ciertas líneas generales en cuanto a su situación económica. En primer lugar, hay notables desequilibrios, especialmente en cuanto a la industria. Por ejemplo, mientras en las cuatro ciudades principales (Bogotá, Medellín, Cali y Barranquilla) sólo se encuentra el 40\% de la población nacional, en ellas se concentran el $65 \%$ de la inversión bruta, el $69.5 \%$ de los establecimientos industriales, el $72 \%$ del empleo industrial, el $70 \%$ del valor agregado industrial, el $70.3 \%$ de la producción bruta industrial y el $72 \%$ de los salarios pagados en la industria (Santana, 1989:180). Esta situación de concentración económica en los centros urbanos se refleja también en los índices de pobreza. En efecto, la pobreza afecta a más del $70 \%$ de la población en el campo y en los pequeños centros poblados, mientras que en las ciudades, alrededor del 31\% de la población es afectada Lora (1990:69). Dado que unos 17 millones de colombianos viven en más de 980 municipios con menos de 100.000 habitantes (es decir, pequeños centros poblados), la frágil situación económica de estos municipios es evidente. La incidencia de la pobreza también tiene marcadas diferencias regionales:

"Las regiones donde la pobreza se encuentra más extendida son, en su orden, Chocó, Córdoba, Sucre y la Orinoquia. En la zona rural de estas regiones la pobreza afecta a más del $88 \%$ de la población. Las regiones de menor incidencia porcentual de la pobreza son Bogotá, Quindío, Risaralda, Caldas y Valle. En ellas, la condición de pobreza absoluta afecta a un poco menos del $29 \%$ de la población".

Estos datos dejan vislumbrar el hecho de que muchos municipios del país comenzaron a poner en marcha la reforma municipal con una base económica bastante precaria. La base económica del municipio condicionó el desarrollo de la reforma municipal de dos maneras. En primera instancia, determinó en gran medida el monto de los ingresos públicos locales obtenido a través de la tributación. Ya se anotó que la ley 14 de 1983 apuntó a fortalecer estos recaudos, especialmente los provenientes de los impuestos Predial, de Industria y Comercio, de Circulación y Tránsito y de otros tributos menores. De hecho, los ingresos municipales propios se han incrementado notablemente en la última década, creciendo en un $65 \%$ entre 1980 y 1987. Los recaudos del impuesto de Industria y Comercio aumentaron en un $85 \%$, y los de Circulación y Tránsito en un 160\% (Zapata: 165-166). Claramente, los municipios que contaban con una fuerte base económica pudieron aprovechar la nueva legislación para generar una cantidad importante de nuevos recursos, tanto para funcionamiento como para inversión. Al mismo tiempo, los municipios más pobres se vieron limitados en su capacidad de producir los recursos previstos por esta norma.

En este contexto, merecen una mención especial los municipios con regalías provenientes de explotaciones petroleras. Con base en la ley 75 de 1986 se determinó que estas regalías serían del $20 \%$, de los cuales 8 puntos serían para la Nación, 9.5 para los departamentos, intendencias y comisarías, y 2.5 para los municipios. Las zonas más beneficiadas por estos ingresos han sido Arauca, Magdalena, Santander, Huila, Meta, Casanare, Antioquia y los 43 municipios con campos petroleros. La importancia de estos recursos para los municipios es obvia cuando se considera que entre 1980 y 1988 las regalías de la provincia pasaron de $\$ 172.5$ millones a $\$ 31.143 .5$ millones (Zapata, 143-161).

En segundo lugar, la base económica del municipio influyó en la reforma. a través de sus efectos indirectos. En especial, condicionó la acción del gobierno municipal por las demandas que creó en áreas sensibles como la salud y el orden público Por ejemplo, un municipio con una precaria base Económica y, por ende, con un alto nivel de pobreza, con frecuencia padece de graves problemas de salud. Para enfrentar esta situación, la administración local debe invertir sus escasos recursos en este rubro. Por otro lado, los municipios con agudos problemas de desempleo, especialmente 
los grandes y medianos, deben afrontar problemas de orden público que también requieren una respuesta del gobierno municipal. De esta manera, municipios con una débil base económica enfrentaron necesidades adicionales que limitaron, aun más, sus posibilidades de acción.

\section{La tradición cívica}

La tradición cívica es el tercer factor estructural que influyó en el resultado de la reforma municipal. Por "tradición cívica” se entiende la presencia o ausencia y el arraigo de los siguientes elementos en la vida pública del municipio: 1) el clientelismo, 2) prácticas de corrupción administrativa, y 3) la Tradición de participación comunitaria. Dado que éste conjunto de elementos tiene una historia muy arraigada en la vida pública de muchos municipios es necesario considerarlo como un factor estructural.

El clientelismo se puede definir como 'las relaciones de intercambio de prestaciones y contraprestaciones entre quienes a falta de recursos (clientelas) garantizan lealtad política hacia terceros en razón de su poder económico, político e influencia social" (Díaz Uribe, 1986: 11). Como tal, es una relación asimétrica por excelencia, y suele ocurrir en ambientes de pobreza donde la gente no tiene otro recurso para solucionar sus problemas. El clientelismo se caracteriza por el uso indebido por parte de un jefe político de recursos que pertenecen al Estado ${ }^{7}$. Aunque las relaciones de clientela están presentes en toda sociedad, en Colombia se han convertido en "el principal soporte de funcionamiento del sistema político”, y como tal en gran medida han sido responsables de la actual crisis de legitimidad del sistema ${ }^{8}$. El clientelismo afectó la reforma municipal principalmente a través de dos modalidades: a) la manipulación del voto, y b) los límites que impuso posteriormente al desarrollo de la reforma.

La manipulación del voto es el aspecto más conocido del clientelismo. Su expresión más común es la compra-venta de votos, aunque recientemente el traslado de votantes ha aumentado en importancia” (OCAMPO y RuIZ, 1991: 172173). La siguiente descripción del "voto amarrado" es, desafortunadamente, generalizable a muchos municipios del país:

"El día de elecciones el "líder" se dirige en vehículo facilitado por su movimiento a recoger los votos adeudados y los electores son llevados al comando electoral en donde son recibidos por los 'guías", quienes se encarga: de acompañarlos hasta la mesa de votación. A los "guías" corresponde garantizar que los votos sean depositados correctamente. Sólo en este momento el sufragante sabe por quién vota" (Díaz Uribe, 43).

En muchos casos, la manipulación del voto resultó en alcaldes supuestamente elegidos "popularmente", pero que en realidad fueron escogidos por los jefes políticos regionales. Como tales, frecuentemente no tenían los conocimientos sobre el municipio requeridos para una buena administración local, ni tenían interés en trabajar para el bien de toda la comunidad. Esas elecciones también afectaron el espíritu cívico de la población y la legitimidad otorgada por ella a las instituciones locales. Como bien lo expresó Alfredo
Manrique Reyes, “¿de qué sirve un alcalde 'electo’ si el mismo pueblo sabe que no fue así y que, por ende, no lo representa? (Manrique Reyes, 1987:557). Pasadas las elecciones, el clientelismo sirvió como elemento para limitar el alcance de la reforma municipal. La lógica del clientelismo se basa en el intercambio entre servicios públicos, maquinaria, puestos, becas, etc., y el apoyo electoral (votos). Acorde con esta lógica, una vez elegido, el alcalde "clientelista” generalmente se limitó a pagar sus "deudas" a los sectores de la comunidad que lo apoyaron en la elección. El bienestar del municipio como un todo se vio perjudicado, y proyectos de largo plazo o de gran cobertura fueron ignorados a cambio de pequeñas obras que dieron algún beneficio inmediato a la clientela del alcalde. De este modo, el clientelismo frenó la reforma en muchos municipios al canalizar los nuevos recursos hacia fines puramente electorales y al limitar el cumplimiento de las nuevas responsabilidades a ciertos aspectos, con predominio del criterio político-partidista.

La corrupción administrativa también forma parte de la tradición cívica de muchos municipios y se vincula estrechamente con el clientelismo. Si este último implica el uso indebido de recursos del Estado para fines políticos, se puede definir la corrupción administrativa como el uso indebido de estos recursos con fines privados. Básicamente, se trata del enriquecimiento ilícito. Aunque cualquier funcionario público puede estar comprometido en la corrupción administrativa, ésta alcanza su mayor grado con los apadrinados por los jefes políticos, ya que su manejo más directo de los recursos locales les facilita el acceso a los dineros municipales. Obviamente, en los municipios donde la corrupción es una práctica generalizada, el desarrollo de la reforma municipal se vio limitado. La tradición de participación comunitaria también condicionó el alcance de la reforma municipal. Uno de los fines enunciados de la reforma era ampliar espacios para la participación comunitaria, dado que:

“...muchas veces la única forma de participar, de hacerse sentir, es la protesta por la falta o las deficiencias en la prestación de los servicios o el rechazo violento al sistema que aparece dando soporte a unas instituciones que han fracasado porque no satisfacen sentidas necesidad?; colectivas ni responden a evidentes urgencias sociales" (Castro, 1984: 36).

De hecho, la reforma contenía nuevos instrumentos de participación a nivel local. Además de la misma Elección Popular de Alcaldes, fueron consagradas la consulta popular, las juntas administradoras locales, la representación de los usuarios en las empresas de servicios públicos y la contratación de organizaciones locales sin ánimo de lucro para la prestación de determinados servicios públicos. Sin embargo, la reforma municipal no tomó en cuenta la débil tradición de participación comunitaria en el país. Aunque en las

7. Francisco Leal Buitrago y Andrés Dávila Ladrón de Guevara dan énfasis a este punto al definir el clientelismo como "la apropiación privada de recursos oficiales con fines políticos". Véase LEAL BUITRAGO y DÁVIla LADRÓN DE GUEVARA (1990: 47).

8. Ibid, pp. 47,31. Este es uno de los principales planteamientos del estudio de LEAL y DÁviLA. 
últimas décadas ha habido intentos para fomentarla a través de organizaciones como la Acción Comunal, la Asociación Nacional de Usuarios Campesinos (ANUC) y, más recientemente, los Consejos Municipales de Rehabilitación pertenecientes al Plan Nacional de Rehabilitación (PNR), muchas veces estas organizaciones se han desprestigiado al verse penetradas por la política y el clientelismo". De tal manera, el municipio que, excepcionalmente contaba con una auténtica tradición de participación comunitaria, se encontraba en condiciones ventajosas para aprovechar los nuevos espacios de participación.

\section{LOS FACTORES COYUNTURALES}

Mientras los factores estructurales establecieron los parámetros dentro de los cuales se desarrolló la reforma municipal, ésta sólo tomó su forma final al ser "filtrada" por los factores coyunturales de cada municipio. Estos factores imprimieron su sello particular sobre la reforma a través de las personalidades de los líderes políticos y las relaciones entre ellos. De especial importancia fueron el alcalde, el Consejo municipal y las relaciones que mantuvo el alcalde con el Consejo, los gamonales, el departamento y la nación.

\section{El alcalde}

Sin duda, el componente más notable de toda la reforma fue la elección popular del alcalde municipal. Como jefe de la administración local, éste se convirtió en el motor principal del desarrollo de la vida municipal. De ahí la incidencia determinante de esta figura en el éxito o fracaso de la reforma. En primer lugar, se debe destacar la personalidad y capacidad de liderazgo del primer alcalde elegido. Un alcalde dinámico y positivo, con una clara capacidad de liderazgo, obviamente tuvo más facilidades para adelantar la reforma que un mandatario carente de estos atributos. Otro elemento que influyó en el éxito del alcalde fue el grado de aceptación de éste en su propia comunidad. Claramente, el alcalde que había vivido y trabajado en el municipio toda su vida y que era conocido y apreciado por sus conciudadanos tenía mayores posibilidades de convocar a la comunidad que el mandatario designado, a dedo por un jefe político regional y elegido a través del sistema de clientelismo. Finalmente, un tercer elemento que afectó la labor del primer alcalde popular fue la existencia de un proyecto político realizable para su municipio. El alcalde que llegó a ejercer el poder con un plan de trabajo serio, ya pensado y elaborado, tenía mayores posibilidades de adelantar una gestión positiva que el mandatario que llegó sin más objetivos que pagar sus deudas políticas. Todo lo anterior sugiere que los municipios con un alcalde dinámico y capaz, con gran aceptación en la comunidad y con un proyecto de gobierno local realizable, partían con las mejores opciones para un relativo éxito en la reforma municipal.

\section{El Consejo municipal}

La Constitución define el Consejo municipal como una corporación administrativa de elección popular integrada por no menos de seis, ni más de veintiún miembros ${ }^{10}$. Formalmente, el Consejo tiene varias funciones; entre ellas, la determinación de la estructura de la administración municipal y la expedición anual del presupuesto de rentas y gastos del municipio. Sin embargo, en la práctica, estas funciones son bastante limitadas. Por un lado, en materia de presupuesto el departamento tiene cierto control sobre la acción del Consejo por ser éste revisado por la oficina de planeación departamental. Por otro lado, el alcalde es quien elabora y presenta el presupuesto al Consejo y este último solamente tiene el poder de aprobarlo o desaprobarlo. El mismo procedimiento se sigue con la presentación de los proyectos de acuerdo. Por esto se ha afirmado que "la verdadera función del Consejo es la de aprobar, desaprobar o reformar las iniciativas del alcalde" (Archer y Esguerra, 1989:125). No obstante, a pesar de lo restringido de sus facultades, el Consejo municipal desempeña un papel decisivo por su capacidad para impulsar u obstaculizar la gestión del alcalde.

Con base en lo anterior, puede afirmarse que dos fueron los elementos coyunturales que determinaron el efecto del Consejo sobre la reforma municipal: 1) la composición política del Consejo y las relaciones subsecuentes y las relaciones subsecuentes con el alcalde, y 2) la seriedad y vocación de servicio de los concejales.

La composición política del Consejo tuvo un efecto notable al robustecer o debilitar el poder del alcalde. Una de las características más generalizadas a nivel local es el fraccionamiento de los partidos políticos. Como señala Eduardo Díaz Uribe:
"A nivel regional lo "político" no se manifiesta en los términos excluyentes de partido liberal o conserva- dor. Esta perspectiva ha perdido importancia. Antes que colectividades políticas nos encontramos con mul- tiplicidad de jefes y movimientos contrincantes... La definición política supone la adhesión, voluntaria o no, de las personas a una de estas fracciones; ser libe- ral o conservador pasa ahora por ser "fulanista" o 'menganista'” (Díaz Uribe, 1986: 37).

De tal manera, lo que cuenta en el Consejo municipal no es tanto la hegemonía de un partido político, ya sea Liberal, Conservador o Comunista, sino el peso de las alianzas entre las múltiples facciones de los partidos. Cuando el Consejo estuvo dominado por una alianza de facciones que apoyaba al alcalde, la gestión de éste se facilitó. Por el contrario, cuando el Consejo fue controlado por facciones políticas opuestas al alcalde su labor se vio dificultada, llegando en algunas ocasiones a paralizarse.

9. Un análisis de la cooptación de las juntas de acción comunal se encuentra en Bruce BAGLEY y Matthew EdEL (1980) 'Popular Mobilization Programs of the National Front: Cooptation and Radicalization”, en R Albert BERRY et. al., Politics of Compromise: Coalition Government in Colombia, New Brunswick, New Jersey: Transaction Books. La historia del auge y subsecuente politización de la ANUC es recontado en Leon ZAMOSC (1986): The Agrarian Question and the Peasant Movement in Colombia: Struggles of the National Peasant Association, 1967-1981. New York: Cambridge University Press.

10. Artículo 312, inciso primero de la Constitución de 1991. En la Constitución de 1886, el número máximo de concejales era de veinte (artículo 196, inciso primero) 
Por otro lado, la seriedad y la vocación de servicio de los concejales también influyeron en el desarrollo de la reforma municipal. Lo importante aquí no era que los concejales fueran profesionales, sino que trabajaran con seriedad y con espíritu cívico de servicio a la comunidad. Es notoria la cantidad de concejales elegidos simplemente como fichas de un jefe político, sin la más mínima intención de aportar ideas o soluciones a los problemas de la comunidad. Obviamente, el alcalde que podía trabajar con concejales idóneos y llegar a acuerdos oportunos para la resolución de problemas locales contaba con mayores posibilidades de adelantar una buena gestión.

\section{Las relaciones interinstitucionales}

Si bien la relación entre el alcalde y el Consejo municipal es clave para el desarrollo de la reforma descentralista, hay otras que también son relevantes. Entre ellas, cabe destacar las existentes entre el alcalde y los gamonales (jefes políticos regionales), el departamento y el Gobierno Central.

Como se anotó al tratar la estructura del clientelismo, los gamonales están presentes en todos los niveles de la política colombiana. Dado que estos jefes muchas veces "controlan" entidades públicas, la relación entre el alcalde y aquéllos es de gran importancia. Así, si un alcalde "cívico" intentaba romper con el esquema de la política tradicional, frecuentemente encontraba que ciertos sectores públicos seguían bajo el control de un gamonal. En estos casos, sólo un hábil manejo de la relación facilito la buena gestión por parte del alcalde. No obstante, aun los alcaldes elegidos con el apoyo del clientelismo tradicional tuvieron que cuidar sus relaciones con los gamonales, especialmente cuando éstos lideraban grupos políticos distintos al del alcalde.

Por otra parte, la relación entre el alcalde y los funcionarios departamentales también fue fundamental. Según la ley, el departamento tiene la función de "prestar asistencia administrativa, técnica y financiera a los municipios, promover su desarrollo y ejercer sobre ellos la tutela que las leyes señalen" ". Aunque no fue determinante, una buena relación entre el alcalde y el gobernador facilito este apoyo. Hay otro aspecto que también merece atención: según la ley 12 de 1986, la ejecución de los planes, programas y proyectos de obras públicas y de desarrollo económico y social de los municipios con población inferior a 100.000 habitantes debe ser vigilada por las oficinas de planeación de los departamentos ${ }^{12}$. En este sentido, el departamento posee un mecanismo que puede ser utilizado -así sea indirectamente- tanto para apoyar como para obstaculizar la labor del alcalde.

Finalmente, hay que destacar la relación entre el alcalde y el Gobierno Central. Por otro lado, la reforma descentralista implicaba la transferencia de varias responsabilidades del nivel central a los gobiernos locales. Sin embargo, aunque el proceso de descentralización significaba la liquidación o reforma de varios organismos del Estado central, definitivamente no implicaba la completa autonomía municipal. Al contrario, muchas de las funciones cumplidas por las entidades desaparecidas fueron transferidas a otros organismos estatales; por ejemplo, al liquidar el Instituto Nacional de Fomento Municipal, Insfopal, sus competencias fueron trasladadas al Ministerio de Obras Públicas y Transporte (Dirección de Agua Potable y Saneamiento Básico), al Ministerio de Salud (Comisión Nacional de Agua Potable), al Departamento Nacional de Planeación y a una entidad privada, el Centro Nacional de Agua, Cenagua. Además, con la reforma municipal se crearon nuevas entidades centrales como 8la Financiera de Desarrollo Territorial, Findeter, encargada de promover el desarrollo local a través de la financiación de proyectos de infraestructura básica. Según esto es claro que la relación entre el alcalde y el Gobierno Central siguió siendo de gran importancia. Además, los ingresos por concepto de transferencias del IVA de los municipios de menos de 100.000 habitantes dependían del informe que sobre sus ingresos por concepto del impuesto predial oportunamente presentaron al Ministerio de Hacienda. En fin, aunque la reforma municipal incrementó la autonomía del gobierno municipal, de ninguna manera terminó con los nexos vitales entre municipio y Gobierno Central. Por ende, las relaciones mantenidas entre el alcalde y las diversas instancias del Estado central cumplieron un papel fundamental en el desarrollo de la reforma municipal.

\section{Tres ejemplos del estudio empírico}

Ni los factores estructurales, ni los coyunturales, determinaron, por sí solos, el resultado de la reforma descentralista en un municipio dado. El relativo éxito o fracaso de la reforma estuvo dado, en última instancia, por la combinación específica de estos factores en cada caso. En los diez municipios de nuestro estudio, la confluencia de estos elementos produjo resultados que van desde muy positivos hasta bastante negativos. A manera de ejemplo, presentamos enseguida tres casos que ayudan a ilustrar los diversos caminos que tomó la reforma municipal en el período de los primeros alcaldes populares.

\section{Un municipio "exitoso"}

El municipio de Cabrera, localizado en la región de Sumapaz, en el departamento de Cundinamarca, fue seleccionado en el estudio por ser pequeño, de agricultura tradicional minifundista y de una clara tradición de izquierda, además de ser uno de los casos donde la violencia política de los años 50 y 60 fue muy notoria. Al iniciarse el primer período de los alcaldes populares, este municipio daba la impresión de carecer de muchos de los elementos conducentes a un buen desarrollo de la reforma. Su infraestructura física y administrativa y su base económica no presentaban un gran desarrollo. Sin embargo, una feliz combinación de elementos, dominados por factores coyunturales benéficos, determinó que la situación de Cabrera se modificara sustancialmente en los primeros dos años de la reforma municipal.

En cuanto a los factores estructurales, tanto la infraestructura municipal como la base económica en 1988, presentaban niveles relativamente bajos. Sin embargo, una fuerte tradición cívica y una relativa equidad en la distribución

11. Artículo 7 del decreto legislativo 1222 de 1986

12. Artículo de ley 12 de 1986. 
de los recursos municipales generaban un escenario algo más propicio para la reforma municipal. La conciencia cívica del municipio se originó en una aguda crisis política que éste afrontó en los años 60, a raíz de la represión armada, y tuvo un catalizador formidable en el Partido Comunista y sus métodos de organización. De esta manera, mientras en 1988 en la mayoría de los municipios del país la tradición de participación comunitaria era prácticamente inexistente, en Cabrera existía con mucha fuerza. Adicionalmente, se presentaban niveles muy bajos de corrupción administrativa. Aunque el clientelismo existía, no era tan excluyente como en muchas localidades y dejaba algún margen para acciones administrativas eficaces.

Por otro lado, la personalidad dinámica y el liderazgo del alcalde encontraron aceptación en la comunidad y ayudaron a movilizar recursos tanto financieros como humanos para modificar la situación inicial del municipio. Específicamente, el alcalde era un joven profesional que, a pesar de no tener ninguna vinculación formal con el establecimiento de los cuadros directivos del Partido Comunista, logró ganarse su confianza. Además, su manejo ágil de los asuntos municipales acercó a la ciudadanía a una administración que tradicionalmente había sido rígida y formal. Aunque inicialmente no tenía un proyecto claro, optó por el pragmatismo, apoyando muchas iniciativas veredales, comunitarias e individuales. En parte, la labor del alcalde se encaminó a proveer maquinaria y algunos recursos financieros a proyectos comunitarios, mientras la gente aportaba la mano de obra y los recursos restantes. Esta estrategia no sólo multiplicó los insumos disponibles, sino que motivó la participación y fiscalización de la ciudadanía en las obras emprendidas. El hecho de que el alcalde no proviniera de los cuadros tradicionales del Partido Comunista local suscito un mayor apoyo de la ciudadanía y, en parte, del Concejo municipal; en esta corporación, el alcalde pudo contar con el respaldo del Partido Comunista y la colaboración de los concejales del Partido Liberal. Así mismo, la posición conciliadora del alcalde y su habilidad de negociación facilitaron las relaciones con el departamento de Cundinamarca. Un último factor que influyó decididamente en el caso de Cabrera fue el Plan Nacional de Rehabilitación. Este contribuyó con recursos financieros significativos, que permitieron construir vías, acueductos, puentes y planteles escolares. En síntesis, Cabrera sirve como ejemplo de un municipio exitoso en los primeros dos años de la reforma municipal. Como muchos municipios del país, no contaba ni con una buena infraestructura ni con una fuerte base económica. Sin embargo, gozaba de una tradición cívica importante. Este factor estructural, combinado con elementos coyunturales tales como un alcalde dinámico, un Concejo colaborador y buenas relaciones interinstitucionales, resultó en un buen manejo de los recursos y responsabilidades previstos en la reforma.

\section{Un municipio "fracaso"}

El municipio de Sincelejo, capital del departamento de Sucre, fue seleccionado en el estudio como ejemplo de una ciudad intermedia de la Costa Atlántica tradicionalmente liberal. Al empezar la experiencia descentralista, la ciudad presentaba unas características muy poco propicias para que la reforma tuviera un efecto positivo. Padecía de estructuras locales bastante débiles que, en combinación con factores coyunturales negativos, determinaron que la reforma municipal no sólo no trajera beneficios a la ciudad, sino que agravara aún más sus problemas endémicos.

$\mathrm{Al}$ iniciar la reforma en 1988, los factores estructurales de la ciudad presentaban un saldo bastante negativo. Por un lado, su infraestructura física tenía serias deficiencias; por ejemplo, la solución del problema de acueducto era inalcanzable, aun si se le hubieran destinado todos los recursos del presupuesto municipal durante varios años. Adicionalmente, la infraestructura administrativa carecía de los elementos básicos para garantizar una gestión aceptable: la altísima inestabilidad laboral y la inexistencia de un control serio del desempeño de los funcionarios hacía que su única responsabilidad fuera jefes políticos a nivel local y regional. La base económica de la ciudad dependía en gran medida de los recursos públicos, y la tradición cívica se caracterizaba por un clientelismo rampante.

A partir de estos factores estructurales desventajosos la presencia de unos factores coyunturales negativos determinaron la profundización de la crisis municipal. El primer alcalde popular provino de la huestes de la política tradicional, con el agravante de haber sido nombrado a través de una alianza preelectoral a nivel departamental. Esta alianza había excluido al grupo del alcalde anterior, lo cual motivó que este dedicara los últimos dos meses de su mandato a gastar y comprometer todos los recursos municipales, especialmente los de transferencia. De esta manera, el nuevo alcalde se vio obligado a pasar su primer año apagando "incendios financieros”, sin posibilidad de ejecutar ninguna acción. Durante el segundo año, la alianza preelectoral inicial se desbarató, dando paso a una reagrupación de fuerzas políticas. La nueva coalición comprometió los recursos del segundo año en acciones clientelistas tendentes a garantizar los resultados electorales de 1990. Como tradicionalmente había sucedido en el departamento de Sucre, la comunidad se mantuvo al margen de este juego "político" y sólo se comprometió a intercambiar los votos por las prebendas correspondientes. En tales circunstancias, el alcalde tampoco tuvo ningún proyecto político. Por otro lado, sus relaciones con el Consejo municipal y con la administración departamental se concentraron en el manejo de las correspondientes cuotas políticas para mantener los equilibrios de los diferentes grupos en la administración pública.

La ciudad de Sincelejo es un ejemplo muy claro de la situación de varios municipios del país, en donde los factores estructurales y coyunturales se combinaron para pervertir la reforma municipal. La falta de una verdadera tradición cívica, en combinación con unas estructuras débiles y factores coyunturales negativos, determinó el resultado final: el empeoramiento de los problemas municipales. En este caso, es claro que la reforma municipal, al desconocer ciertas particularidades locales, especialmente el clientelismo, incidió en la promoción de una mayor ineficiencia administrativa y en un aumento en el despilfarro y la corrupción, través de los nuevos recursos y responsabilidades. 


\section{Un municipio "neutral"}

Un municipio de Ubaté, ubicado en el noreste de Cundinamarca, fue seleccionado por ser de tamaño pequeño, con un desarrollo agropecuario de tipo comercial y una clara tradición conservadora. Al iniciar el primer período de alcaldes elegidos popularmente, el municipio contaba con algunos factores estructurales que hacían prever que la reforma produciría efectos muy positivos. Sin embargo, estos elementos favorables se combinaron con otros factores estructurales y coyunturales, de manera que los dos primeros años de reforma pasaron sin que se observaran cambios sustanciales en la gestión municipal.

Por un lado, el municipio contaba con una infraestructura física y administrativa relativamente adecuada, en donde, pro ejemplo, el cubrimiento de servicios públicos estaba por encima del promedio nacional y departamental. Además, Ubaté gozaba de una base económica relativamente sólida, fundamentada en la ganadería lechera, la agricultura, los servicios y el apoyo a la minería de carbón en municipios vecinos. Sin embargo, a pesar de estos factores positivos, la tradición cívica de Ubaté estaba minada por la política tradicional, con fuertes dosis de clientelismo, corrupción administrativa y escasa participación comunitaria. El claro predominio de una facción del Partido Conservador había reducido la competencia política a su mínima expresión. Aunque en los últimos años el liberalismo había aumentado su representación, su profunda división interna no ofreció ningún incentivo que propiciara la unidad conservadora. Es así como un candidato minoritario de este último partido ganó la alcaldía, con consecuencias importantes para el desarrollo de la reforma municipal.

De otra parte, los factores coyunturales no fueron muy alentadores para la reforma descentralista. Por un lado, el alcalde propendió la exclusión de los demás grupos políticos, incluyendo los de su propio partido. Esto le llevó a perder el apoyo tanto del Concejo municipal como de la clase política local. Adicionalmente, su compromiso con un grupo de alcaldes del departamento para destituir al gobernador generó la obstaculización total de su administración por parte del gobierno departamental. Esto llevó, por ejemplo, a que sus iniciativas no sólo no encontraran eco en las secretarías de la Gobernación de Cundinamarca, sino a que el gobierno departamental llegara a entrabarle el funcionamiento de la administración municipal. Paralelamente, la capacidad de liderazgo del alcalde fue muy baja frente a los grupos políticos, aunque ésta se manifestó más positivamente frente a la ciudadanía. Estos factores coyunturales no hubieran sido definitivos si el proceso político local hubiera estado dominado por una organización comunitaria fuerte. Sin embargo, como ya se dijo, el clientelismo tradicional era el pilar sobre el cual estaba construida la actividad política local. Al perder el apoyo de la clase política, el alcalde se quedó solo y no pudo realizar ningún cambio importante en la vida municipal.

Ubaté es un ejemplo de muchos otros municipios del país en donde la reforma descentralista no significó una gran modificación en la vida local. A pesar de contar con una infraestructura municipal y una base económica aceptables, otros factores como la tradición cívica, la personalidad y liderazgo del alcalde, y sus relaciones inter-institucionales, determinaron que la reforma municipal quedara estancada.

\section{CONCLUSIÓN}

Los tres casos anteriores sirven como ejemplos del complejo proceso iniciado por la reforma municipal. Demuestran que no se puede generalizar sobre las bondades o perjuicios de este proceso. También ilustran nuestra explicación teórica sobre los diversos impactos de la reforma la cual plantea que los resultados de ésta en cada municipio dependían de una combinación compleja de factores estructurales y coyunturales. Al utilizar tal explicación, se evitan conclusiones simplistas sobre un proceso que ha desencadenado sin duda unos cambios muy favorables para el desarrollo local, pero que también ha tenido efectos negativos para la vida municipal. A raíz de este análisis, se destaca la gran variedad de resultados y el potencial de una reforma que no se puede circunscribir a una interpretación jurídica. El esquema teórico presentado en este capítulo es apenas un comienzo; sin embargo, puede servir como punto de partida de futuras investigaciones sobre la vida municipal del país. Todavía quedan muchos interrogantes, que sólo se podrán resolver a través de estudios empíricos. Por ejemplo, ¿cuál es la base económica actual de los más de 1.000 municipios en términos de ingresos y desempleo? ¿Qué tan generalizadas son las prácticas clientelistas en el país? Y a nivel, más teórico, ¿bajo qué condiciones priman los factores estructurales y bajo cuales los coyunturales, en la determinación del relativo éxito o fracaso de una gestión municipal? Finalmente, este estudio tiene una implicación eminentemente práctica. Sugiere que en cualquier reforma descentralista no se puede ignorar el efecto de factores como la infraestructura municipal, la base económica, y la tradición cívica de un municipio. Mientras el legislador difícilmente puede controlar los factores coyunturales, sí puede obrar tomando en cuenta el efecto de los elementos estructurales en el desarrollo de la descentralización. Ignorar este aspecto equivale a condenar a muchos municipios colombianos a la profundización de sus crisis y a llevar a sus habitantes a un mayor desencanto con el gobierno local lo cual, en últimas, sólo sirve para profundizar la crisis de legitimidad del Estado.

\section{BIBLIOGRAFÍA}

ArCHer, R. y Esguerra, G. (1989): "Las instituciones departamentales y municipales frente a la descentralización y la elección popular de alcaldes", en VÁsQuez de URRUTia, P. (comp.) (1989): La democracia en blanco y negro: Colombia en los años ocbenta. Bogotá: Ediciones Uniandes -Departamento de Ciencia Política- Cerec.

Bagley, B. y Edel, M. (1980) 'Popular Mobilization Programs of the National Front: Cooptation and Radicalization", en BERRY, R.A. et. al., Politics of Compromise: Coalition Government in Colombia, New Brunswick, New Jersey: Transaction Books.

BlanQuer, J. M. (1991): "Descentralización y desarrollo de los servicios públicos”, en Blanquer, J.-M. y FAJARDo, D.: La descentralización en Colombia: estudios y propuestas. Bogotá: 
IFEA- Universidad Nacional de Colombia, Centro de Estudios Sociales

CASTRO, J. (1984): La democracia local: ideas para un nuevo régimen departamental y municipal. Bogotá, Editorial Oveja Negra.

DíAz URIBE, E. (1986): El clientelismo en Colombia: un estudio exploratorio. Bogotá: El Áncora Editores.

Dugas, J., OCAMPO, A., ORjuEla, L. J. y RuIZ, G. (1992): Los caminos de la descentralización: diversidad y retos de la transformación municipal. Bogotá: Universidad de Los Andes.

GarcíA, J. I. (1991): “Antioquia, premio del agua”, en El Tiempo, octubre 2 .

Leal Buitrago, F. y Dávila Ladrón de Guevara, A. (1990): Clientelismo: el sistema político y su expresión regional. Bogotá: Tercer Mundo - Universidad Nacional / Instituto de Estudios Politices y Relaciones Internacionales,

LORA, E. T. (1990): "La estructura de la economía colombiana", en LORA, E. y OCAMPO, J. A. (Coordinadores): Introducción a la macroeconomía colombiana. Bogotá: Tercer Mundo EditoresFedesarrollo.

MANRIQUE REYES, A. (1987): "Descentralización y modernización del Estado: un proceso a medias", en Revista Javeriana, No. 537, Bogotá (agosto)

OCAMPO, A. y RuIZ, G. "Las elecciones de alcaldes: avances y retrocesos”, en Sánchez David, R. (comp.) (1991): Los nuevos retos electorales. Bogotá: Cerec-Universidad de los Andes, Departamento de Ciencia Política.

Santana, P. R. (1989): Los movimientos sociales en Colombia Bogotá: Ediciones Foro Nacional por Colombia.

ZamosC, L. (1986): The Agrarian Question and the Peasant Movement in Colombia: Struggles of the National Peasant Association, 1967-1981. New York: Cambridge University Press.

ZAPATA, J.G. (1991): "Evaluación de las principales leyes de descentralización económica en la década de los 80", en GAITÁN, P. et.al., Comunidad, alcaldes y recursos fiscales. Bogotá: Fescol.

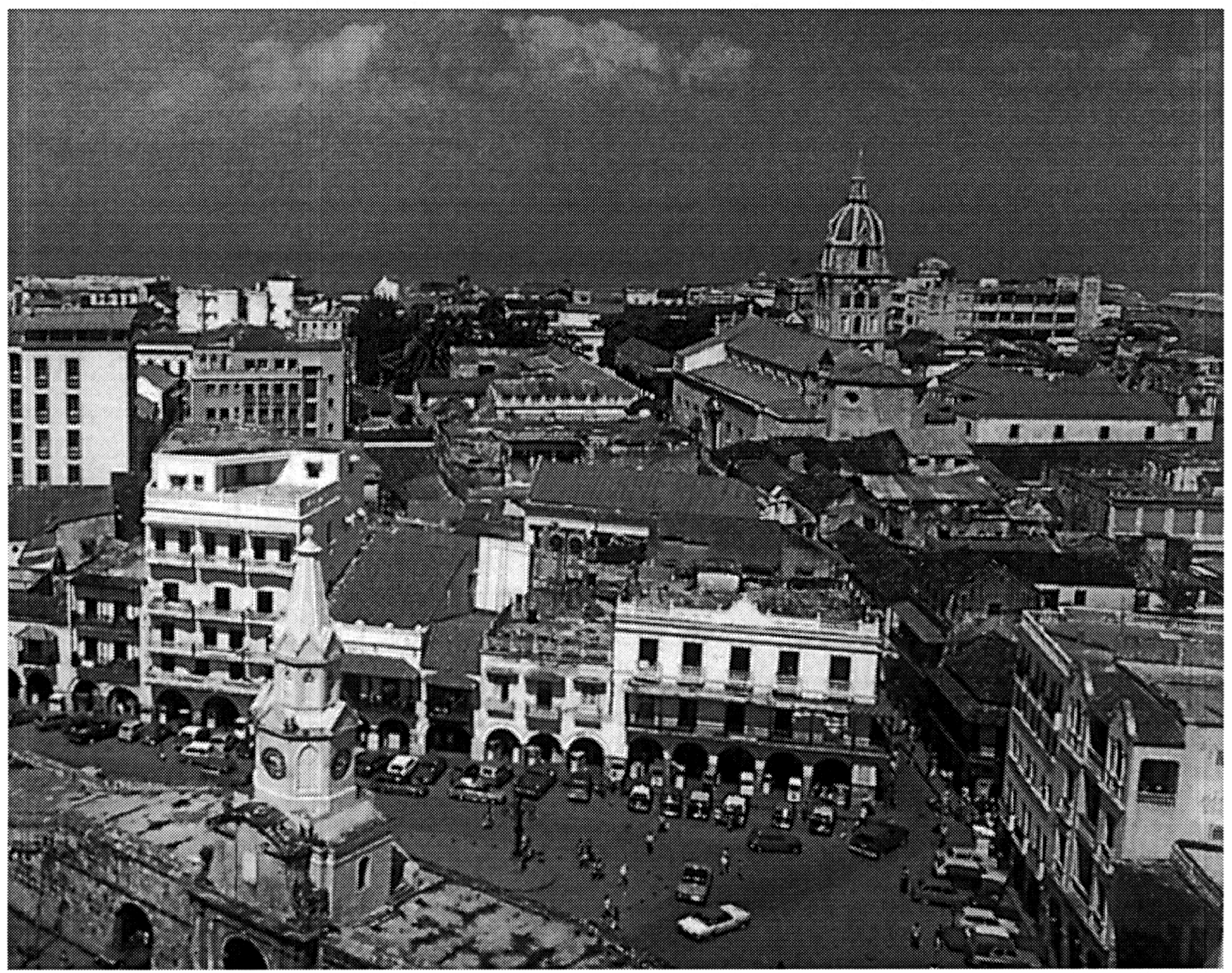

